\title{
Heavy baryon spectroscopy
}

\author{
Rudolf N. Faustov ${ }^{1}$ and Vladimir $O$. Galkin ${ }^{1}$ \\ ${ }^{1}$ Institute of Cybernetics and Informatics in Education, FRC CSC RAS, Moscow, Russia
}

\begin{abstract}
Masses of heavy baryons are calculated in the relativistic quarkdiquark picture. Obtained results are in good agreement with available experimental data including recent measurements by the LHCb Collaboration. Possible quantum numbers of excited heavy baryon states are discussed.
\end{abstract}

\section{Introduction}

Recently significant experimental progress has been achieved in studying heavy baryon spectroscopy. Many new heavy baryon states have been observed. The main contribution was made by the LHCb Collaboration. Thus last year the amplitude analysis of the decay $\Lambda_{b}^{0} \rightarrow D^{0} p \pi^{-}$was performed in the region of the phase space containing $D^{0} p$ resonant contributions which revealed three $\Lambda_{c}$ excited states and allowed to measure precisely their masses and decay widths [1]: the $\Lambda_{c}(2880)^{+}$with the preferred spin $J=5 / 2$; the new state $\Lambda_{c}(2860)^{+}$ with quantum numbers $J^{P}=3 / 2^{+}$, its parity was measured relative to that of the $\Lambda_{c}(2880)^{+}$; the $\Lambda_{c}(2940)^{+}$with the most likely spin-parity assignment $J^{P}=3 / 2^{-}$but other solutions with spins from $1 / 2$ to $7 / 2$ were not excluded. Then five new, narrow excited $\Omega_{c}$ states decaying to $\Xi_{c}^{+} K^{-}$were observed [2]: the $\Omega_{c}(3000)^{0}, \Omega_{c}(3050)^{0}, \Omega_{c}(3066)^{0}, \Omega_{c}(3090)^{0}$, and $\Omega_{c}(3119)^{0}$. These states were later confirmed by Belle [3]. Soon the discovery of the long-awaited doubly charmed baryon $\Xi_{c c}^{++}$was reported [4]. This year the new $\Xi_{b}(6227)^{-}$resonance was observed as a peak in both the $\Lambda_{b}^{0} K^{-}$and $\Xi_{b}^{0} \pi^{-}$invariant mass spectra [5]. Finally, the first observation of two structures $\Sigma(6097)^{ \pm}$consistent with resonances in the final states $\Lambda_{b}^{0} \pi^{-}$and $\Lambda_{b}^{0} \pi^{+}$was reported by the $\mathrm{LHCb}[6]$.

In this talk we compare these new data with the predictions of the relativistic quarkdiquark model of heavy baryons [7-9].

\section{Relativistic quark-diquark model of heavy baryons}

Our approach is based on the relativistic quark-diquark picture and the quasipotential equation. The interaction of two quarks in a diquark and the quark-diquark interaction in a baryon are described by the diquark wave function $\Psi_{d}$ of the bound quark-quark state and by the baryon wave function $\Psi_{B}$ of the bound quark-diquark state respectively. These wave functions satisfy the relativistic quasipotential equation of the Schrödinger type [7]

$$
\left(\frac{b^{2}(M)}{2 \mu_{R}}-\frac{\mathbf{p}^{2}}{2 \mu_{R}}\right) \Psi_{d, B}(\mathbf{p})=\int \frac{d^{3} q}{(2 \pi)^{3}} V(\mathbf{p}, \mathbf{q} ; M) \Psi_{d, B}(\mathbf{q}),
$$


where $\mu_{R}$ is the relativistic reduced mass, $b^{2}(M)$ is the center-of-mass relative momentum squared on the mass shell, $\mathbf{p}, \mathbf{q}$ are the off-mass-shell relative momenta, and $M$ is the bound state mass (diquark or baryon).

The kernel $V(\mathbf{p}, \mathbf{q} ; M)$ in Eq. (1) is the quasipotential operator of the quark-quark or quarkdiquark interaction which is constructed with the help of the off-mass-shell scattering amplitude, projected onto the positive energy states. We assume that the effective interaction is the sum of the usual one-gluon exchange term and the mixture of long-range vector and scalar linear confining potentials, where the vector confining potential contains the Pauli term. The vertex of the diquark-gluon interaction takes into account the diquark internal structure and effectively smears the Coulomb-like interaction. The corresponding form factor is expressed as an overlap integral of the diquark wave functions. Explicit expressions for the quasipotentials of the quark-quark interaction in a diquark and quark-diquark interaction in a baryon can be found in [8]. All parameters of the model were fixed previously from considerations of meson properties and are kept fixed in the baryon spectrum calculations.

The quark-diquark picture of heavy baryons reduces a very complicated relativistic threebody problem to a significantly more simpler two step two-body calculation. First we determine the properties of diquarks. We consider a diquark to be a composite $\left(q q^{\prime}\right)$ system. Thus diquark in our approach is not a point-like object. Its interaction with gluons is smeared by the form factor expressed through the overlap integral of diquark wave functions. These form factors enter the diquark-gluon interaction and effectively take diquark structure into account $[8,9]$. Note that the ground state diquark composed from quarks with different flavours can be both in scalar and axial vector state, while the ground state diquarks composed from quarks of the same flavour can be only in the axial vector state due to the Pauli principle. Solving the quasipotential equation numerically we calculate the masses, determine the diquark wave functions and use them for evaluation of the diquark form factors. Only ground-state scalar and axial vector diquarks are considered for heavy baryons. While both qround-state as well as orbital and radial excitations of heavy diquarks are necessary for doubly heavy baryons, since the lowest excitations of such baryons originate from the excitations of the doubly heavy diquark.

Next we calculate the masses of heavy baryons in the quark-diquark picture $[8,9]$. The heavy baryon is considered as a bound state of a heavy-quark and light-diquark. All excitations are assumed to occur between heavy quark and light diquark. On the other hand, the doubly heavy baryon is considered as a bound state of a light-quark and heavy-diquark. Both excitations in the quark-diquark system and excitations of the heavy diquark are taken into account. It is important to note that such approach predicts significantly less excited states of baryons compared to a genuine three-quark picture. We do not expand the potential of the quark-diquark interaction either in $p / m_{q, Q}$ or in $p / m_{d}$ and treat both diquark and quark fully relativistically.

\section{Heavy baryons}

The calculated masses of heavy baryons are given in Tabs. 1-5. In the first column we show the baryon total isospin $I$, spin $J$ and parity $P$. The second column lists the quark-diquark state. The next three columns refer to the charm and the last there columns to the bottom baryons. There we first give our prediction for the mass, then available experimental data [10]: baryon status and measured mass. The charm and bottom baryon states recently discovered by the LHCb Collaboration $[1,2,4-6]$ are marked as new.

From Tabs. 1, 2 we see that the $\Lambda_{c}(2765)$ (or $\Sigma_{c}(2765)$ ), if it is indeed the $\Lambda_{c}$ state, can be interpreted in our model as the first radial $(2 S)$ excitation of the $\Lambda_{c}$. If instead it is the $\Sigma_{c}$ state, then it can be identified as its first orbital excitation $(1 P)$ with $J=\frac{3}{2}^{-}$(see Tab. 2). The 
Table 1. Masses of the $\Lambda_{Q}(Q=c, b)$ heavy baryons (in $\mathrm{MeV}$ )

\begin{tabular}{|c|c|c|c|c|c|c|c|}
\hline \multirow[b]{2}{*}{$I\left(J^{P}\right)$} & \multirow[b]{2}{*}{$Q d$ state } & \multicolumn{3}{|c|}{$Q=c$} & \multicolumn{3}{|c|}{$Q=b$} \\
\hline & & $M$ & status & $M^{\exp }$ & $M$ & status & $M^{\exp }$ \\
\hline \multirow[t]{6}{*}{$0\left(\frac{1}{2}^{+}\right)$} & $1 S$ & 2286 & $* * * *$ & $2286.46(14)$ & 5620 & $* * *$ & $5619.51(23)$ \\
\hline & $2 S$ & 2769 & $*$ & $2766.6(2.4) ?$ & 6089 & & \\
\hline & $3 S$ & 3130 & & & 6455 & & \\
\hline & $4 S$ & 3437 & & & 6756 & & \\
\hline & $5 S$ & 3715 & & & 7015 & & \\
\hline & $6 S$ & 3973 & & & 7256 & & \\
\hline \multirow[t]{5}{*}{$0\left(\frac{1}{2}^{-}\right)$} & $1 P$ & 2598 & $* * *$ & $2592.25(28)$ & 5930 & $* * *$ & $5912.11(26)$ \\
\hline & $2 P$ & 2983 & $* * *$ & $2944.8\left(\begin{array}{l}1.4 \\
15\end{array}\right) ?$ & 6326 & & \\
\hline & $3 P$ & 3303 & & & 6645 & & \\
\hline & $4 P$ & 3588 & & & 6917 & & \\
\hline & $5 P$ & 3852 & & & 7157 & & \\
\hline \multirow[t]{5}{*}{$0\left(\frac{3}{2}^{-}\right)$} & $1 P$ & 2627 & $* * *$ & $2628.1(6)$ & 5942 & $* * *$ & $5919.81(23)$ \\
\hline & $2 P$ & 3005 & & & 6333 & & \\
\hline & $3 P$ & 3322 & & & 6651 & & \\
\hline & $4 P$ & 3606 & & & 6922 & & \\
\hline & $5 P$ & 3869 & & & 7171 & & \\
\hline \multirow[t]{4}{*}{$0\left(\frac{3}{2}^{+}\right)$} & $1 D$ & 2874 & new & $2856.1 .3\left(\begin{array}{c}2.3 \\
59\end{array}\right)$ & 6190 & & \\
\hline & $2 D$ & 3189 & & & 6526 & & \\
\hline & $3 D$ & 3480 & & & 6811 & & \\
\hline & $4 D$ & 3747 & & & 7060 & & \\
\hline \multirow[t]{4}{*}{$0\left(\frac{5}{2}^{+}\right)$} & $1 D$ & 2880 & $* * *$ & $2881.75(35)$ & 6196 & & \\
\hline & $2 D$ & 3209 & & & 6531 & & \\
\hline & $3 D$ & 3500 & & & 6814 & & \\
\hline & $4 D$ & 3767 & & & 7063 & & \\
\hline \multirow[t]{4}{*}{$0\left(\frac{5}{2}^{-}\right)$} & $1 F$ & 3097 & & & 6408 & & \\
\hline & $2 F$ & 3375 & & & 6705 & & \\
\hline & $3 F$ & 3646 & & & 6964 & & \\
\hline & $4 F$ & 3900 & & & 7196 & & \\
\hline \multirow[t]{4}{*}{$0\left(\frac{7}{2}^{-}\right)$} & $1 F$ & 3078 & & & 6411 & & \\
\hline & $2 F$ & 3393 & & & 6708 & & \\
\hline & $3 F$ & 3667 & & & 6966 & & \\
\hline & $4 F$ & 3922 & & & 7197 & & \\
\hline \multirow[t]{2}{*}{$0\left(\frac{7}{2}^{+}\right)$} & $1 G$ & 3270 & & & 6598 & & \\
\hline & $2 G$ & 3546 & & & 6867 & & \\
\hline \multirow[t]{2}{*}{$0\left(\frac{9}{2}^{+}\right)$} & $1 G$ & 3284 & & & 6599 & & \\
\hline & $2 G$ & 3564 & & & 6868 & & \\
\hline $0\left(\frac{9}{2}^{-}\right)$ & $1 H$ & 3444 & & & 6767 & & \\
\hline $0\left(\frac{11}{2}^{-}\right)$ & $1 H$ & 3460 & & & 6766 & & \\
\hline
\end{tabular}


Table 2. Masses of the $\Sigma_{Q}(Q=c, b)$ heavy baryons (in $\mathrm{MeV}$ )

\begin{tabular}{|c|c|c|c|c|c|c|c|}
\hline \multirow[b]{2}{*}{$I\left(J^{P}\right)$} & \multirow[b]{2}{*}{$Q d$ state } & \multicolumn{3}{|c|}{$Q=c$} & \multicolumn{3}{|c|}{$Q=b$} \\
\hline & & $M$ & status & $M^{\exp }$ & $M$ & status & $M^{\exp }$ \\
\hline \multirow[t]{5}{*}{$1\left(\frac{1}{2}^{+}\right)$} & $1 S$ & 2443 & $* * * *$ & $2453.76(18)$ & 5808 & $* * *$ & $5807.8(2.7)$ \\
\hline & $2 S$ & 2901 & & & 6213 & & \\
\hline & $3 S$ & 3271 & & & 6575 & & \\
\hline & $4 S$ & 3581 & & & 6869 & & \\
\hline & $5 S$ & 3861 & & & 7124 & & \\
\hline \multirow[t]{5}{*}{$1\left(\frac{3}{2}^{+}\right)$} & $1 S$ & 2519 & $* * *$ & $2518.0(5)$ & 5834 & $* * *$ & $5829.0(3.4)$ \\
\hline & $2 S$ & 2936 & $* * *$ & $2939.3\left(\begin{array}{l}1.4 \\
15\end{array}\right) ?$ & 6226 & & \\
\hline & $3 S$ & 3293 & & & 6583 & & \\
\hline & $4 S$ & 3598 & & & 6876 & & \\
\hline & $5 S$ & 3873 & & & 7129 & & \\
\hline \multirow[t]{8}{*}{$1\left(\frac{1}{2}^{-}\right)$} & $1 P$ & 2799 & $* * *$ & $2802\left(\begin{array}{l}4 \\
7\end{array}\right)$ & 6101 & & \\
\hline & $2 P$ & 3172 & & & 6440 & & \\
\hline & $3 P$ & 3488 & & & 6756 & & \\
\hline & $4 P$ & 3770 & & & 7024 & & \\
\hline & $1 P$ & 2713 & & & 6095 & & \\
\hline & $2 P$ & 3125 & & & 6430 & & \\
\hline & $3 P$ & 3455 & & & 6742 & & \\
\hline & $4 P$ & 3743 & & & 7008 & & \\
\hline \multirow[t]{8}{*}{$1\left(\frac{3}{2}^{-}\right)$} & $1 P$ & 2798 & *** & $2802\left(\begin{array}{l}4 \\
7\end{array}\right)$ & 6096 & new & $6095.8(1.8)$ \\
\hline & $2 P$ & 3172 & & & 6430 & & \\
\hline & $3 P$ & 3486 & & & 6742 & & \\
\hline & $4 P$ & 3768 & & & 7009 & & \\
\hline & $1 P$ & 2773 & $*$ & $2766.6(2.4) ?$ & 6087 & & \\
\hline & $2 P$ & 3151 & & & 6423 & & \\
\hline & $3 P$ & 3469 & & & 6736 & & \\
\hline & $4 P$ & 3753 & & & 7003 & & \\
\hline \multirow[t]{4}{*}{$1\left(\frac{5}{2}^{-}\right)$} & $1 P$ & 2789 & & & 6084 & & \\
\hline & $2 P$ & 3161 & & & 6421 & & \\
\hline & $3 P$ & 3475 & & & 6732 & & \\
\hline & $4 P$ & 3757 & & & 6999 & & \\
\hline \multirow[t]{2}{*}{$1\left(\frac{1}{2}^{+}\right)$} & $1 D$ & 3041 & & & 6311 & & \\
\hline & $2 D$ & 3370 & & & 6636 & & \\
\hline \multirow[t]{4}{*}{$1\left(\frac{3}{2}^{+}\right)$} & $1 D$ & 3043 & & & 6326 & & \\
\hline & $2 D$ & 3366 & & & 6647 & & \\
\hline & $1 D$ & 3040 & & & 6285 & & \\
\hline & $2 D$ & 3364 & & & 6612 & & \\
\hline \multirow[t]{4}{*}{$1\left(\frac{5}{2}^{+}\right)$} & $1 D$ & 3038 & & & 6284 & & \\
\hline & $2 D$ & 3365 & & & 6612 & & \\
\hline & $1 D$ & 3023 & & & 6270 & & \\
\hline & $2 D$ & 3349 & & & 6598 & & \\
\hline \multirow[t]{2}{*}{$1\left(\frac{7}{2}^{+}\right)$} & $1 D$ & 3013 & & & 6260 & & \\
\hline & $2 D$ & 3342 & & & 6590 & & \\
\hline
\end{tabular}


Table 3. Masses of the $\Xi_{Q}(Q=c, b)$ heavy baryons with the scalar diquark (in MeV)

\begin{tabular}{|c|c|c|c|c|c|c|c|}
\hline \multirow[b]{2}{*}{$I\left(J^{P}\right)$} & \multirow[b]{2}{*}{$Q d$ state } & \multicolumn{3}{|c|}{$Q=c$} & \multicolumn{3}{|c|}{$Q=b$} \\
\hline & & $M$ & status & $M^{\exp }$ & $M$ & status & $M^{\exp }$ \\
\hline \multirow{5}{*}{$\frac{1}{2}\left(\frac{1}{2}^{+}\right)$} & $1 S$ & 2476 & $* * *$ & $2470.88\left(\begin{array}{l}34 \\
80\end{array}\right)$ & 5803 & $* * *$ & $5790.5(2.7)$ \\
\hline & $2 S$ & 2959 & & & 6266 & & \\
\hline & $3 S$ & 3323 & & & 6601 & & \\
\hline & $4 S$ & 3632 & & & 6913 & & \\
\hline & $5 S$ & 3909 & & & 7165 & & \\
\hline \multirow{5}{*}{$\frac{1}{2}\left(\frac{1}{2}^{-}\right)$} & $1 P$ & 2792 & $* * *$ & $2792.8(1.2)$ & 6120 & & \\
\hline & $2 P$ & 3179 & & & 6496 & & \\
\hline & $3 P$ & 3500 & & & 6805 & & \\
\hline & $4 P$ & 3785 & & & 7068 & & \\
\hline & $5 P$ & 4048 & & & 7302 & & \\
\hline \multirow[t]{5}{*}{$\frac{1}{2}\left(\frac{3}{2}^{-}\right)$} & $1 P$ & 2819 & $* * *$ & $2820.22(32)$ & 6130 & & \\
\hline & $2 P$ & 3201 & & & 6502 & & \\
\hline & $3 P$ & 3519 & & & 6810 & & \\
\hline & $4 P$ & 3804 & & & 7073 & & \\
\hline & $5 P$ & 4066 & & & 7306 & & \\
\hline \multirow[t]{4}{*}{$\frac{1}{2}\left(\frac{3}{2}^{+}\right)$} & $1 D$ & 3059 & $* * *$ & $3055.9(0.4)$ & 6366 & & \\
\hline & $2 D$ & 3388 & & & 6690 & & \\
\hline & $3 D$ & 3678 & & & 6966 & & \\
\hline & $4 D$ & 3945 & & & 7208 & & \\
\hline \multirow[t]{4}{*}{$\frac{1}{2}\left(\frac{5}{2}^{+}\right)$} & $1 D$ & 3076 & $*$ & $3079.9(1.4)$ & 6373 & & \\
\hline & $2 D$ & 3407 & & & 6696 & & \\
\hline & $3 D$ & 3699 & & & 6970 & & \\
\hline & $4 D$ & 3965 & & & 7212 & & \\
\hline
\end{tabular}

$\Lambda_{c}(2880)$ baryon corresponds to the second orbital excitation $(2 D)$ with $J=\frac{5}{2}^{+}$in accord with the LHCb analysis [1]. The other charmed baryon, denoted as $\Lambda_{c}(2940)$, probably has $I=0$, since it was discovered in the $p D^{0}$ mass spectrum and not observed in $p D^{+}$channel, but $I=1$ is not ruled out [10]. If it is really the $\Lambda_{c}$ state, then it could be both an orbitally and radially excited $(2 P)$ state with $J=\frac{1}{2}^{-}$, whose mass is predicted to be about $40 \mathrm{MeV}$ heavier. A better agreement with experiment (within few $\mathrm{MeV}$ ) is achieved, if the $\Lambda_{c}(2940)$ is interpreted as the first radial excitation $(2 S)$ of the $\Sigma_{c}$ with $J=\frac{3}{2}^{+}$. The $\Sigma_{c}(2800)$ can be identified with one of the first orbital $(1 P)$ excitations of the $\Sigma_{c}$ with $J=\frac{1}{2}^{-}$or $\frac{3}{2}^{-}$which have very close masses compatible with experimental value within errors (see Tab. 2). The new state $\Lambda_{c}(2860)$ with quantum numbers $\frac{3}{2}^{+}$[1] can be well interpreted as second orbital excitation ( $2 D$ state). In the bottom sector the $\Lambda_{b}(5912)$ and $\Lambda_{b}(5920)$ correspond to the first orbitally excited $(1 P)$ states with $\frac{1}{2}^{-}$and $\frac{3}{2}^{-}$, respectively. The new $\Sigma_{b}(6097)$ state [6] can be the first orbital excitation $(1 P)$ with quantum numbers $\frac{3}{2}^{-}$.

In the $\Xi_{Q}$ baryon sector as we see from Tabs. 3,4 the $\Xi_{c}(2790)$ and $\Xi_{c}(2815)$ can be assigned to the first orbital $(1 P)$ excitations of the $\Xi_{c}$ containing a scalar diquark with $J=\frac{1}{2}^{-}$ and $J=\frac{3}{2}^{-}$, respectively. On the other hand, the charmed baryon $\Xi_{c}(2930)$ can be considered as either the $J=\frac{1}{2}^{-}, J=\frac{3}{2}^{-}$or $J=\frac{5}{2}^{-}$state (all these states are predicted to have close masses) corresponding to the first orbital $(1 P)$ excitations of the $\Xi_{c}^{\prime}$ with an axial vector diquark. While the $\Xi_{c}(2980)$ can be viewed as the first radial $(2 S)$ excitation with $J=\frac{1}{2}^{+}$ 
Table 4. Masses of the $\Xi_{Q}(Q=c, b)$ heavy baryons with the axial vector diquark (in MeV)

\begin{tabular}{|c|c|c|c|c|c|c|c|}
\hline \multirow[b]{2}{*}{$I\left(J^{P}\right)$} & \multirow[b]{2}{*}{$Q d$ state } & \multicolumn{3}{|c|}{$Q=c$} & \multicolumn{3}{|c|}{$Q=b$} \\
\hline & & $M$ & status & $M^{\exp }$ & $M$ & status & $M^{\text {exp }}$ \\
\hline \multirow{5}{*}{$\frac{1}{2}\left(\frac{1}{2}^{+}\right)$} & $1 S$ & 2579 & $* * *$ & $2577.9(2.9)$ & 5936 & $* * *$ & $5935.02(5)$ \\
\hline & $2 S$ & 2983 & & $2971.4(3.3)$ & 6329 & & \\
\hline & $3 S$ & 3377 & & & 6687 & & \\
\hline & $4 S$ & 3695 & & & 6978 & & \\
\hline & $5 S$ & 3978 & & & 7229 & & \\
\hline \multirow{5}{*}{$\frac{1}{2}\left(\frac{3}{2}^{+}\right)$} & $1 S$ & 2649 & $* * *$ & $2645.9(0.5)$ & 5963 & $* * *$ & $5955.33(13)$ \\
\hline & $2 S$ & 3026 & & & 6342 & & \\
\hline & $3 S$ & 3396 & & & 6695 & & \\
\hline & $4 S$ & 3709 & & & 6984 & & \\
\hline & $5 S$ & 3989 & & & 7234 & & \\
\hline \multirow{8}{*}{$\frac{1}{2}\left(\frac{1}{2}^{-}\right)$} & $1 P$ & 2936 & $*$ & 2931(6) & 6233 & & \\
\hline & $2 P$ & 3313 & & & 6611 & & \\
\hline & $3 P$ & 3630 & & & 6915 & & \\
\hline & $4 P$ & 3912 & & & 7174 & & \\
\hline & $1 P$ & 2854 & & & 6227 & new & $6226.9(2.1)$ \\
\hline & $2 P$ & 3267 & & & 6604 & & \\
\hline & $3 P$ & 3598 & & & 6906 & & \\
\hline & $4 P$ & 3887 & & & 7164 & & \\
\hline \multirow[t]{8}{*}{$\frac{1}{2}\left(\frac{3}{2}^{-}\right)$} & $1 P$ & 2935 & $*$ & 2931(6) & 6234 & & \\
\hline & $2 P$ & 3311 & & & 6605 & & \\
\hline & $3 P$ & 3628 & & & 6905 & & \\
\hline & $4 P$ & 3911 & & & 7163 & & \\
\hline & $1 P$ & 2912 & & & 6224 & new & $6226.9(2.1)$ \\
\hline & $2 P$ & 3293 & & & 6598 & & \\
\hline & $3 P$ & 3613 & & & 6900 & & \\
\hline & $4 P$ & 3898 & & & 7159 & & \\
\hline \multirow[t]{4}{*}{$\frac{1}{2}\left(\frac{5}{2}^{-}\right)$} & $1 P$ & 2929 & $*$ & 2931(6) & 6226 & new & $6226.9(2.1)$ \\
\hline & $2 P$ & 3303 & & & 6596 & & \\
\hline & $3 P$ & 3619 & & & 6897 & & \\
\hline & $4 P$ & 3902 & & & 7156 & & \\
\hline$\frac{1}{2}\left(\frac{1}{2}^{+}\right)$ & $1 D$ & 3163 & & & 6447 & & \\
\hline \multirow[t]{2}{*}{$\frac{1}{2}\left(\frac{3}{2}^{+}\right)$} & $1 D$ & 3167 & & & 6459 & & \\
\hline & $1 D$ & 3160 & & & 6431 & & \\
\hline \multirow[t]{2}{*}{$\frac{1}{2}\left(\frac{5}{2}^{+}\right)$} & $1 D$ & 3166 & & & 6432 & & \\
\hline & $1 D$ & 3153 & & & 6420 & & \\
\hline$\frac{1}{2}\left(\frac{7}{2}^{+}\right)$ & $1 D$ & 3147 & $*$ & $3122.9(1.3)$ & 6414 & & \\
\hline
\end{tabular}

of the $\Xi_{c}^{\prime}$, the $\Xi_{c}(3055)$ and $\Xi_{c}(3080)$ baryons can be interpreted as a second orbital $(2 D)$ excitations of the $\Xi_{c}$ containing a scalar diquark with $J=\frac{3}{2}^{+}$and $J=\frac{5}{2}^{+}$, and the $\Xi_{c}(3123)$ can be viewed as the corresponding $(2 D)$ excitation of the $\Xi_{c}^{\prime}$ with $J=\frac{7}{2}^{+}$. The recently observed excited bottom baryon $\Xi_{b}(6227)^{-}$[5] can be one of the first radially excited states $(1 P)$ of the $\Xi_{b}^{\prime}$ baryon with the axial vector diquark and quantum numbers $\frac{1}{2}^{-}, \frac{3}{2}^{-}, \frac{5}{2}^{-}$which are predicted to have very close masses. 
Table 5. Masses of the $\Omega_{Q}(Q=c, b)$ heavy baryons (in $\mathrm{MeV}$ )

\begin{tabular}{|c|c|c|c|c|c|c|c|}
\hline \multirow[b]{2}{*}{$I\left(J^{P}\right)$} & \multirow[b]{2}{*}{$Q d$ state } & \multicolumn{3}{|c|}{$Q=c$} & \multicolumn{3}{|c|}{$Q=b$} \\
\hline & & $M$ & status & $M^{\exp }$ & $M$ & status & $M^{\exp }$ \\
\hline \multirow[t]{5}{*}{$0\left(\frac{1}{2}^{+}\right)$} & $1 S$ & 2698 & $* * *$ & $2695.2(1.7)$ & 6064 & $* * *$ & 6046.4(1.9) \\
\hline & $2 S$ & 3088 & new & $3090.2\left(\begin{array}{l}7 \\
8\end{array}\right)$ & 6450 & & \\
\hline & $3 S$ & 3489 & & & 6804 & & \\
\hline & $4 S$ & 3814 & & & 7091 & & \\
\hline & $5 S$ & 4102 & & & 7338 & & \\
\hline \multirow[t]{5}{*}{$0\left(\frac{3}{2}^{+}\right)$} & $1 S$ & 2768 & $* * *$ & $2765.9(2.0)$ & 6088 & & \\
\hline & $2 S$ & 3123 & new & $3119.1\left(\begin{array}{l}1.0 \\
1.1\end{array}\right)$ & 6461 & & \\
\hline & $3 S$ & 3510 & & & 6811 & & \\
\hline & $4 S$ & 3830 & & & 7096 & & \\
\hline & $5 S$ & 4114 & & & 7343 & & \\
\hline \multirow[t]{8}{*}{$0\left(\frac{1}{2}^{-}\right)$} & $1 P$ & 3055 & & & 6339 & & \\
\hline & $2 P$ & 3435 & & & 6710 & & \\
\hline & $3 P$ & 3754 & & & 7009 & & \\
\hline & $4 P$ & 4037 & & & 7265 & & \\
\hline & $1 P$ & 2966 & & & 6330 & & \\
\hline & $2 P$ & 3384 & & & 6706 & & \\
\hline & $3 P$ & 3717 & & & 7003 & & \\
\hline & $4 P$ & 4009 & & & 7257 & & \\
\hline \multirow[t]{8}{*}{$0\left(\frac{3}{2}^{-}\right)$} & $1 P$ & 3054 & new & $3065.6\left(\frac{6}{7}\right)$ & 6340 & & \\
\hline & $2 P$ & 3433 & & & 6705 & & \\
\hline & $3 P$ & 3752 & & & 7002 & & \\
\hline & $4 P$ & 4036 & & & 7258 & & \\
\hline & $1 P$ & 3029 & new & $3000.4\left(\frac{4}{6}\right)$ & 6331 & & \\
\hline & $2 P$ & 3415 & & & 6699 & & \\
\hline & $3 P$ & 3737 & & & 6998 & & \\
\hline & $4 P$ & 4023 & & & 7250 & & \\
\hline \multirow[t]{4}{*}{$0\left(\frac{5}{2}^{-}\right)$} & $1 P$ & 3051 & new & $3050.2\left(\frac{4}{5}\right)$ & 6334 & & \\
\hline & $2 P$ & 3427 & & & 6700 & & \\
\hline & $3 P$ & 3744 & & & 6996 & & \\
\hline & $4 P$ & 4028 & & & 7251 & & \\
\hline $0\left(\frac{1}{2}^{+}\right)$ & $1 D$ & 3287 & & & 6540 & & \\
\hline \multirow[t]{2}{*}{$0\left(\frac{3}{2}^{+}\right)$} & $1 D$ & 3298 & & & 6549 & & \\
\hline & $1 D$ & 3282 & & & 6530 & & \\
\hline \multirow[t]{2}{*}{$0\left(\frac{5}{2}^{+}\right)$} & $1 D$ & 3297 & & & 6529 & & \\
\hline & $1 D$ & 3286 & & & 6520 & & \\
\hline $0\left(\frac{7}{2}^{+}\right)$ & $1 D$ & 3283 & & & 6517 & & \\
\hline $0\left(\frac{3}{2}^{-}\right)$ & $1 F$ & 3533 & & & 6763 & & \\
\hline
\end{tabular}

Masses of the $\Omega_{c}$ and $\Omega_{b}$ baryons are given in Tab. 5. The ground state (1S) masses were predicted [7] before experimental discovery and agree well with measured values. Recently observed [2] five new, narrow excited $\Omega_{c}$ are also in accord with our predictions. Three lighter states $\Omega_{c}(3000)^{0}, \Omega_{c}(3050)^{0}$ and $\Omega_{c}(3066)^{0}$ are well described as first orbital (1P) excitations with $J=\frac{3}{2}^{-}, \frac{5}{2}^{-}$and $\frac{3}{2}^{-}$, respectively. These states are expected to be narrow. The remaining $1 P$ states with $\frac{1}{2}^{-}$are expected to be broad and thus can escape detection. The small peak 


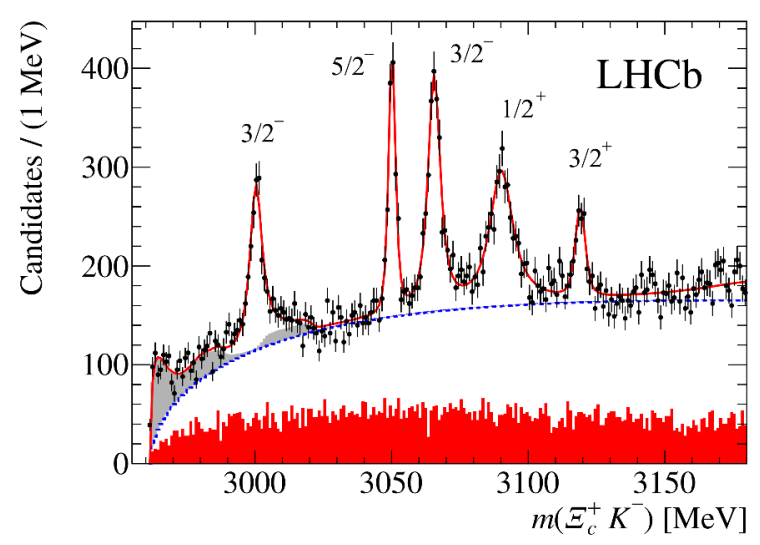

Figure 1. Proposed assignment of spins and parities of excited $\Omega_{c}$ states observed by LHCb Collaboration

in the low end of $\Xi_{c}^{+} K^{-}$mass distribution (see Fig. 1) can correspond to $\frac{1}{2}^{-}$state with the predicted mass $2966 \mathrm{MeV}$ (see Tab. 5). The remaining two heavier states $\Omega_{c}(3090)^{0}$ and $\Omega_{c}(3119)^{0}$ are naturally described as first radial $(2 S)$ excitations with quantum numbers $\frac{1}{2}^{+}$ and $\frac{3}{2}^{+}$, respectively. Their predicted masses coincide with the measured ones within a few $\mathrm{MeV}$. The proposed assignment of spins and parities of excited $\Omega_{c}$ states observed by LHCb Collaboration is given in Fig. 1. In Tab. 6 we compare different quark model (QM), QCD sum rules (QCD SR), lattice QCD predictions and available experimental data for the masses of the $\Omega_{c}$ states.

Table 6. Comparison of theoretical predictions for the masses of the $\Omega_{c}$ states

\begin{tabular}{|c|c|c|c|c|c|c|c|}
\hline $\begin{array}{c}\text { State } \\
n L, J^{P}\end{array}$ & $\begin{array}{c}\text { our [8] } \\
\text { RQM }\end{array}$ & $\begin{array}{l}{[11]} \\
\text { QM }\end{array}$ & $\begin{array}{l}{[12]} \\
\mathrm{QM} \\
\end{array}$ & $\begin{array}{c}{[14]} \\
\text { lattice }\end{array}$ & $\begin{array}{c}{[13]} \\
\text { lattice }\end{array}$ & $\begin{array}{c}{[15]} \\
\text { QCD SR }\end{array}$ & $\begin{array}{l}\text { Experiment. } \\
\text { PDG+LHCb }\end{array}$ \\
\hline $1 S, \frac{1}{2}^{+}$ & 2698 & 2718 & 2695 & $2648(28)$ & $2695(28)$ & $2685(123)$ & $2695.2(1.7)$ \\
\hline $2 S, \frac{1}{2}^{+}$ & 3088 & 3152 & 3100 & $3294(73)$ & & $3066(138)$ & $3090.2\left(\begin{array}{l}7 \\
8\end{array}\right)$ \\
\hline $1 S, \frac{3}{2}^{+}$ & 2768 & 2776 & 2767 & $2709(32)$ & 2781(25) & 2769(89) & $2765.9(2.0)$ \\
\hline $2 S, \frac{3}{2}^{+}$ & 3123 & 3190 & 3126 & $3355(92)$ & & $3119(114)$ & $3119.1\left(\begin{array}{l}1.0 \\
1.1\end{array}\right)$ \\
\hline $1 P, \frac{1}{2}^{-}$ & 2966 & 2977 & 3028 & 2995(46) & $3015(45)$ & & \\
\hline $1 P, \frac{1}{2}^{-}$ & 3055 & 2990 & 3011 & & & & \\
\hline $1 P, \frac{3}{2}^{-}$ & 3054 & 2986 & 2976 & $3016(69)$ & & & $3065.6\left(\begin{array}{l}6 \\
7\end{array}\right)$ \\
\hline $1 P, \frac{3}{2}^{-}$ & 3029 & 2994 & 2993 & & & & $3000.4\left(\begin{array}{l}4 \\
6\end{array}\right)$ \\
\hline $1 P, \frac{5}{2}^{-}$ & 3051 & 3014 & 2947 & & & & $3050.2\left(\begin{array}{c}4 \\
5\end{array}\right)$ \\
\hline
\end{tabular}

\section{Doubly heavy baryons}

Mass spectra of doubly heavy baryons was calculated in the light-quark-heavy-diquark picture in [9]. The light quark was treated completely relativistically, while the expansion in the inverse heavy quark mass was used. Tab. 7 shows the $\Xi_{c c}$ mass spectrum. Excitaions inside doubly heavy diquark and light-quark-heavy-diquark bound systems are taken into account. We use the notations $\left(n_{d} L n_{q} l\right) J^{P}$, where we first show the radial quantum number of the diquark $\left(n_{d}=1,2,3 \ldots\right)$ and its orbital momentum by a capital letter $(L=S, P, D \ldots)$, then 
Table 7. Mass spectrum of $\Xi_{c c}$ baryons (in $\mathrm{GeV}$ )

\begin{tabular}{cccccc}
\hline State & \multicolumn{2}{c}{ Mass } & State & \multicolumn{2}{c}{ Mass } \\
$\left(n_{d} L n_{q} l\right) J^{P}$ & our & {$[16]$} & $\left(n_{d} L n_{q} l\right) J^{P}$ & our & {$[16]$} \\
\hline$(1 S 1 s) \frac{1}{2}^{+}$ & 3.620 & 3.478 & $(1 P 1 s) \frac{1}{2}^{-}$ & 3.838 & 3.702 \\
$(1 S 1 s) \frac{3}{2}^{+}$ & 3.727 & 3.61 & $(1 P 1 s) \frac{3}{2}^{-}$ & 3.959 & 3.834 \\
$(1 S 1 p) \frac{1}{2}^{-}$ & 4.053 & 3.927 & $(2 S 1 s) \frac{1}{2}^{+}$ & 3.910 & 3.812 \\
$(1 S 1 p) \frac{3}{2}^{-}$ & 4.101 & 4.039 & $(2 S 1 s) \frac{3}{2}^{+}$ & 4.027 & 3.944 \\
$(1 S 1 p) \frac{1}{2}^{-}$ & 4.136 & 4.052 & $(2 P 1 s) \frac{1}{2}^{-}$ & 4.085 & 3.972 \\
$(1 S 1 p) \frac{5}{2}^{-}$ & 4.155 & 4.047 & $(2 P 1 s) \frac{3}{2}^{-}$ & 4.197 & 4.104 \\
$(1 S 1 p) \frac{3}{2}^{-}$ & 4.196 & 4.034 & $(3 S 1 s) \frac{1}{2}^{+}$ & 4.154 & 4.072 \\
\hline
\end{tabular}

Table 8. Mass spectrum of ground states of doubly heavy baryons (in $\mathrm{GeV}$ ). $\{Q Q\}$ denotes the diquark in the axial vector state and $[Q Q]$ denotes diquark in the scalar state

\begin{tabular}{cccccccccc}
\hline Baryon Quark & $J^{P}$ & our & {$[16]$} & {$[17]$} & {$[18]$} & {$[19]$} & {$[11]$} & {$[20]$} \\
& content & & {$[9]$} & & & & & & \\
\hline$\Xi_{c c}$ & $\{c c\} q$ & $\frac{1}{2}^{+}$ & 3.620 & 3.478 & 3.66 & 3.69 & 3.510 & 3.676 & $3.627(12)$ \\
$\Xi_{c c}^{*}$ & $\{c c\}_{q}$ & $\frac{3}{2}^{+}$ & 3.727 & 3.61 & 3.74 & & 3.548 & 3.753 & $3.690(12)$ \\
$\Omega_{c c}$ & $\{c c\} s$ & $\frac{1}{2}^{+}$ & 3.778 & 3.59 & 3.74 & 3.86 & 3.719 & 3.815 & \\
$\Omega_{c c}^{*}$ & $\{c c\}_{s}$ & $\frac{3}{2}^{+}$ & 3.872 & 3.69 & 3.826 & & 3.746 & 3.876 & \\
$\Xi_{b b}$ & $\{b b\}_{q}$ & $\frac{1}{2}^{+}$ & 10.202 & 10.093 & 10.34 & 10.16 & 10.130 & 10.340 & $10.162(12)$ \\
$\Xi_{b b}^{*}$ & $\{b b\}_{q}$ & $\frac{3}{2}^{+}$ & 10.237 & 10.133 & 10.37 & & 10.144 & 10.367 & $10.184(12)$ \\
$\Omega_{b b}$ & $\{b b\}_{s}$ & $\frac{1}{2}^{+}$ & 10.359 & 10.18 & 10.37 & 10.34 & 10.422 & 10.454 & \\
$\Omega_{b b}^{*}$ & $\{b b\}_{s}$ & $\frac{3}{2}^{+}$ & 10.389 & 10.20 & 10.40 & & 10.432 & 10.486 & \\
$\Xi_{c b}$ & $\{c b\}_{q}$ & $\frac{1}{2}^{+}$ & 6.933 & 6.82 & 7.04 & 6.96 & 6.792 & 7.011 & $6.914(13)$ \\
$\Xi_{c b}^{\prime}$ & {$[c b] q$} & $\frac{1}{2}^{+}$ & 6.963 & 6.85 & 6.99 & & 6.825 & 7.047 & $6.933(12)$ \\
$\Xi_{c b}^{*}$ & $\{c b\}_{q}$ & $\frac{3}{2}^{+}$ & 6.980 & 6.90 & 7.06 & & 6.827 & 7.074 & $6.969(14)$ \\
$\Omega_{c b}$ & $\{c b\}_{s}$ & $\frac{1}{2}^{+}$ & 7.088 & 6.91 & 7.09 & 7.13 & 6.999 & 7.136 & \\
$\Omega_{c b}^{\prime}$ & {$[c b] s$} & $\frac{1}{2}^{+}$ & 7.116 & 6.93 & 7.06 & & 7.022 & 7.165 & \\
$\Omega_{c b}^{*}$ & $\{c b\}_{s}$ & $\frac{3}{2}^{+}$ & 7.130 & 6.99 & 7.12 & & 7.024 & 7.187 & \\
\hline & & & & & & & & & \\
\hline
\end{tabular}

the radial quantum number of the light quark $\left(n_{q}=1,2,3 \ldots\right)$ and its orbital momentum by a lowercase letter $(l=s, p, d \ldots)$, and at the end the total angular momentum $J$ and parity $P$ of the baryon. In Tab. 8 we compare different theoretical predictions for the ground state masses of the doubly heavy baryons. Our prediction (2002) for the mass of the $\Xi_{c c}$ baryon [9] excellently agrees with its mass recently measured (2017) by the LHCb Collaboration [6]:

$$
M^{\exp }\left(\Xi_{c c}^{++}\right)=3621.40 \pm 0.72 \pm 0.27 \pm 0.14 \mathrm{MeV}
$$

\section{Conclusions}

Recent observations of excited charm and bottom baryons confirm predictions of the relativistic heavy-quark-light-diquark model of heavy baryons [7, 8]. The new state $\Lambda_{c}(2860)$ is in accord with the predicted $1 D$ - state with $J^{P}=\frac{3}{2}^{+}$. The experimentally preferred quantum numbers $J^{P}=\frac{5}{2}^{+}$of $\Lambda_{c}(2880)$ agree with our assignment of this state to $1 D$ - state with $J^{P}=\frac{5}{2}^{+}$. The $\Lambda_{b}(5912)$ and $\Lambda_{b}(5920)$ are well described as the first orbitally excited $(1 P)$ states with $\frac{1}{2}^{-}$and $\frac{3}{2}^{-}$, respectively. The new $\Sigma_{b}(6097)$ state can be the first orbital excitation 
$(1 P)$ with quantum numbers $\frac{3}{2}^{-}$. The recently observed excited bottom baryon $\Xi_{b}(6227)^{-}$can be one of the first radially excited states $(1 P)$ of the $\Xi_{b}^{\prime}$ baryon with the axial vector diquark and quantum numbers $\frac{1}{2}^{-}, \frac{3}{2}^{-}, \frac{5}{2}^{-}$which are predicted to have very close masses. Observation of five new narrow $\Omega_{c}$ states in the mass range $3000-3200 \mathrm{MeV}$ agrees with our prediction of orbitally excited $1 P$-states and radially excited $2 S$-states in this mass region: $\Omega_{c}(3000)$, $\Omega_{c}(3066), \Omega_{c}(3050)$ can be $1 P$-states with $J^{P}=\frac{3}{2}^{-}, \frac{3}{2}^{-}, \frac{5}{2}^{-}$while $\Omega_{c}(3090)$ and $\Omega_{c}(3119)$ states are most likely the first radially excited $2 S$ states with $J^{P}=\frac{1}{2}^{+}, \frac{3}{2}^{+}$.

In the doubly heavy baryon sector, the mass of recently observed $\Xi_{c c}^{++}$baryon is in excellent agreement with our prediction made more than 15 years ago [9]. Masses of ground state doubly charm baryons are predicted to be in 3.5 - 3.9 GeV range. Masses of ground state doubly bottom baryons are predicted to be in $10.1-10.5 \mathrm{GeV}$ range. Masses of ground state bottom-charm baryons are predicted to be in $6.8-7.2 \mathrm{GeV}$ range. Rich spectra of narrow excited states below strong decay thresholds are expected. We strongly encourage experimenters to search for new excited states of heavy baryons and especially for doubly heavy baryons.

Acknowledgments. We are grateful to I. Belyaev, D. Ebert, M. Ivanov, M. Karliner and A. Martin for valuable discussions and support. We thank the organizers of XXIV International Baldin Seminar on High Energy Physics Problems.

\section{References}

[1] R. Aaij et al. [LHCb Collaboration], JHEP 1705, 030 (2017)

[2] R. Aaij et al. [LHCb Collaboration], Phys. Rev. Lett. 118(18), 182001 (2017)

[3] J. Yelton et al. [Belle Collaboration], Phys. Rev. D 97(5), 051102 (2018)

[4] R. Aaij et al. [LHCb Collaboration], Phys. Rev. Lett. 119(11), 112001 (2017)

[5] R. Aaij et al. [LHCb Collaboration], Phys. Rev. Lett. 121(7), 072002 (2018)

[6] R. Aaij et al. [LHCb Collaboration], arXiv:1809.07752 [hep-ex]

[7] D. Ebert, R. N. Faustov and V. O. Galkin, Phys. Rev. D 72, 034026 (2005). Phys. Lett. B 659, 612 (2008)

[8] D. Ebert, R. N. Faustov and V. O. Galkin, Phys. Rev. D 84, 014025 (2011)

[9] D. Ebert, R. N. Faustov, V. O. Galkin and A. P. Martynenko, Phys. Rev. D 66, 014008 (2002)

[10] M. Tanabashi et al. [Particle Data Group], Phys. Rev. D 98(3), 030001 (2018)

[11] W. Roberts and M. Pervin, Int. J. Mod. Phys. A 23, 2817 (2008)

[12] Z. Shah, K. Thakkar, A. K. Rai and P. C. Vinodkumar, Chin. Phys. C 40(12), 123102 (2016)

[13] Y. C. Chen et al. [TWQCD Collaboration], Phys. Lett. B 767, 193 (2017)

[14] P. Pérez-Rubio, S. Collins and G. S. Bali, Phys. Rev. D 92(3), 034504 (2015)

[15] S. S. Agaev, K. Azizi and H. Sundu, Eur. Phys. J. C 77(6), 395 (2017)

[16] S. S. Gershtein, V. V. Kiselev, A. K. Likhoded and A. I. Onishchenko, Phys. Rev. D 62, 054021 (2000)

[17] R. Roncaglia, D. B. Lichtenberg and E. Predazzi, Phys. Rev. D 52, 1722 (1995)

[18] I. M. Narodetskii and M. A. Trusov, Phys. Atom. Nucl. 65, 917 (2002) [Yad. Fiz. 65, 949 (2002)]

[19] A. P. Martynenko, Phys. Lett. B 663, 317 (2008)

[20] M. Karliner and J. L. Rosner, Phys. Rev. D 90(9), 094007 (2014) 